To appear in the proceedings of the conference "The Origins of Stars and Planets: the VLT View",

J. Alves, and M. McCaughrean (Eds.); http://www.eso.org/gen-fac/meetings/starplanet2001

\title{
Extra-Solar Planetary Systems in the VLT Era
}

\author{
Michel Mayor and Nuno C. Santos \\ Geneva Observatory, 51 Ch. des Maillettes, CH-1290 Sauverny, Switzerland
}

\begin{abstract}
Radial Velocity surveys have revealed 63 exoplanets ( $\mathrm{M} \sin i<10 \mathrm{M}_{\mathrm{Jup}}$ ) and 6 multi-planetary systems (status on the 4th of April 2001). Distributions of orbital elements already have been given some constraints on the formation of planetary systems. Derived from recent high resolution spectroscopy studies, the impressive role of the stellar metallicity on the giant planet formation has been revealed. The chemical composition of the molecular cloud is probably the key parameter to form giant planets. However some evidences exist showing the possibility of accretion of matter in the stellar outer convective zone.
\end{abstract}

\section{Introduction}

Following the discovery in 1995 of the planet orbiting $51 \mathrm{Peg}$ [20], we have witnessed a complete revolution in the field of extra-solar planets. More than 60 other exo-planets were unveiled since then, and are giving us the opportunity to reconsider the theories dealing with planetary formation and evolution.

In particular, the information gathered from the radial-velocity surveys can be interpreted as fossil traces of the planet formation and evolution processes. By looking at the planetary orbital characteristics, like the distribution of eccentricities and periods, or to the planetary masses, we are facing a lot of questions on planetary formation. Furthermore, other evidences are coming from the planet host stars themselves, namely by the fact that stars with planets are very metalrich. The new results are showing that planet formation is not as simple as we thought. In the next Sects. we will summarize the latest results on this field.

\section{The Period Distribution}

One of the most interesting problems that appeared after the first planets were discovered as to do with their proximity from their host stars. Several mechanisms have been proposed to explain this fact. Current results show that in situ formation is very unlikely [2], and we have to invoke inward migration, either due to gravitational interaction with the disk [7], [13] or with other companions [25] to explain the observed orbital periods.

Although still strongly biased for the long period systems, the period distribution of the extra-solar planetary companions can already tell us something about the planetary formation and evolution processes. This is particularly true for the short period systems, for which the biases are not so important. In Fig. 1 


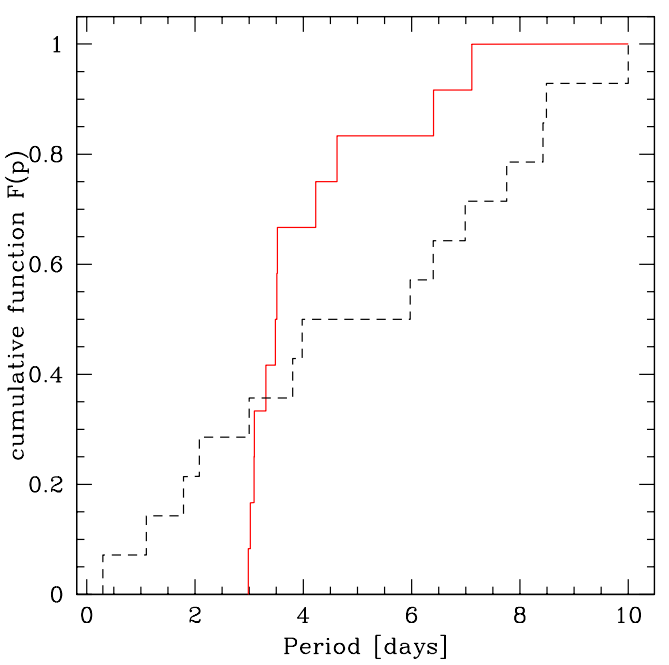

Fig. 1. Cumulative distributions of periods smaller than 10 days for planetary (solid line) and stellar companions (dashed line) to solar type dwarfs.

we present the orbital period cumulative function for stars with planetary companions $\left(\mathrm{M}<10 \mathrm{M}_{\mathrm{Jup}}\right)$ with $\mathrm{P}<10$ days, as well as for solar type binary stars in the same period regime. The most impressive feature in the diagram is the clear cutoff of the planet distribution for periods shorter than $\sim 3$ days. To explain this distribution, several ideas have been presented, invoking e.g. a magnetospheric central cavity of the accretion disk, tidal interaction with the host star, Roche lobe overflow by the young inflated giant planet, or evaporation (see [27] and references therein).

\section{The Mass Distribution}

Another important clue concerning the nature of the now discovered planetary systems comes from their mass distribution. Clearly, given that the radialvelocity technique is more sensitive to massive companions, we could expect to find more "massive" planets when compared to their less massive counterparts. However, a look at the mass distribution tells us exactly the contrary (Fig. 2).

Several conclusions may be taken from the plots. First, the gap in the distribution, separating low mass stellar companions from the lower mass planets (often called the "brown dwarf desert") represents a strong evidence that these two populations are the result of different formation processes. Second, we can see that the planetary mass distribution has a sharp cutoff for masses lower than $\sim 10 \mathrm{M}_{\text {Jup }}[27]$. This limit is clearly not related to the D-burning mass limit of $13 \mathrm{M}_{\mathrm{Jup}}$. As was recently shown [12], and contrary to some recent suggestions [10] 

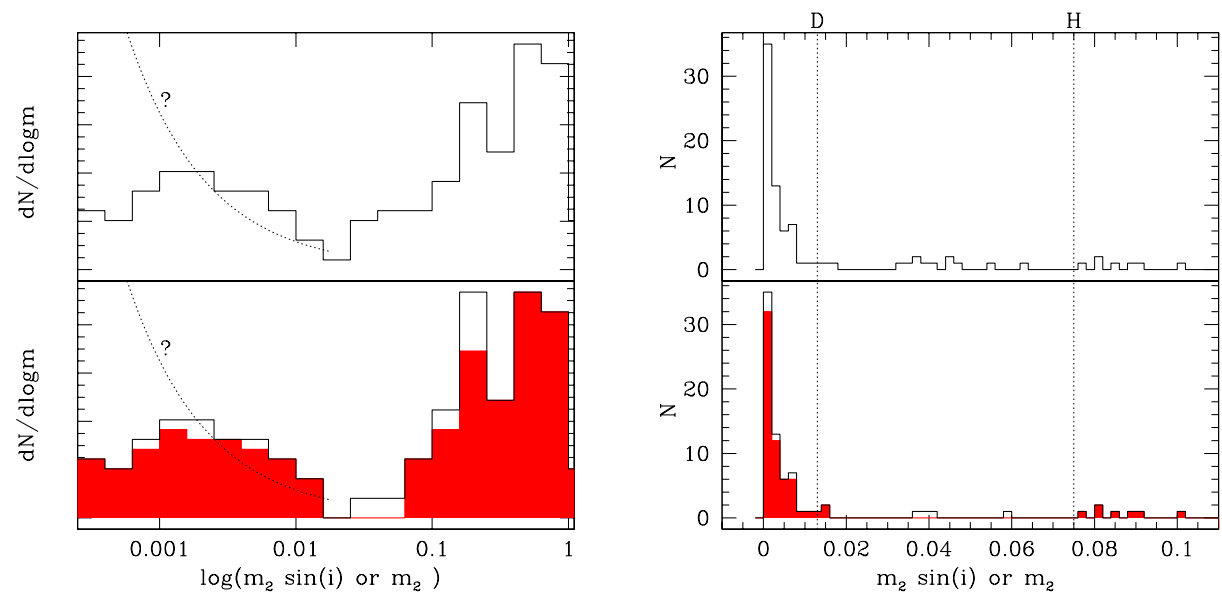

Fig. 2. Mass function of companions to solar-type stars in $\log$ (left) and linear (right) scales. The dotted vertical lines indicate the H- and D-burning limits. Top: $m_{2} \sin i$. Bottom: composite histograms of $m_{2}$ (open part) and $m_{2} \sin i$ (if $\sin i$ not known).

(easily refuted by statistical arguments [9], [24]), this conclusion is not an artifact of the fact that for most of the targets we only have minimum masses, but a real upper limit for the mass of the planetary companions discovered so far.

\section{The Distribution of Eccentricities}

One of the most enigmatic results to date is illustrated in Fig. 3. A look at the figure shows that there are no clear differences between the eccentricity distributions of planetary and stellar binary systems. How then can this be fit into the "traditional" picture of a planet forming in a disk? For masses lower than $\sim 20 M_{\text {Jup }}$, it has been shown that the interaction with a disk has the effect of damping the eccentricity [22]. This suggests that other processes, like the interaction between planets in a multiple system, or the influence of a distant stellar companion, may play an important role in defining the "final" orbital configuration. In this respect, one particularly interesting case of very high eccentricity amongst the planetary companions is the planet around HD 80606 [21] (Fig. 4).

Although still not clear, however, a close inspection of the Fig. 3, permits to find a few differences between stellar and planetary companions eccentricities. For example, for periods in the range of 10 to 30 days (clearly outside the circularization period by tidal interaction with the star), there are a few stars with planets having very low eccentricity, while no stellar binaries are present in this region. On the other hand, for the very short period systems, we can see some planetary companions with eccentricities higher than those found for "stars". This facts may be telling us that different formation and evolution processes took place: for example, the former group may be seen as a sign for formation 


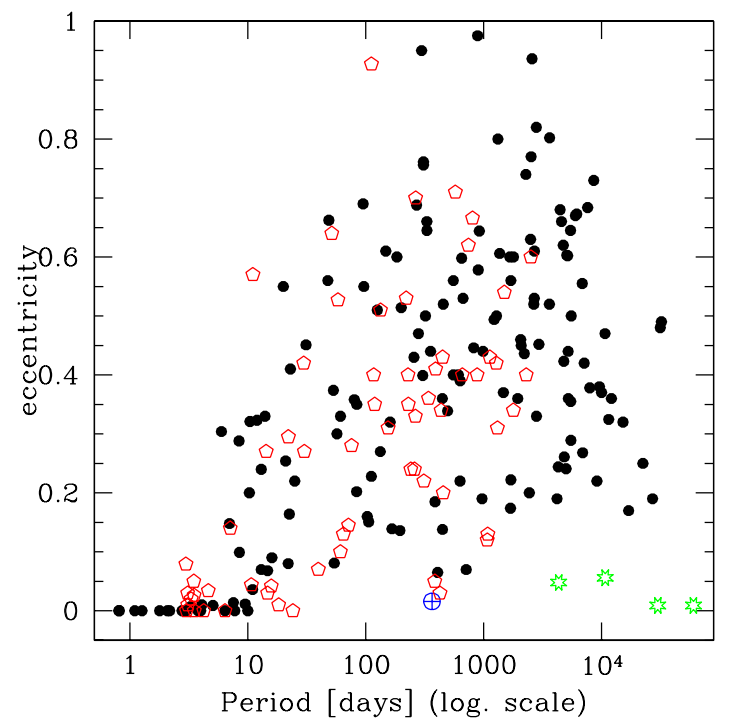

Fig. 3. The $e-\log P$ diagram for planetary (open pentagons) and stellar companions (filled circles) to solar type field dwarfs. Starred symbols represent the giant planets of our Solar System, while the "earth" symbol represents our planet.

in a disk, and the latter one as an evidence of the influence of a longer period companion on the eccentricity (cf. case of HD 217107 [6] and HD 83443 [18]).

\section{Multi-planetary Systems}

Another possible source of information about the formation of giant planets may came from the multi-planetary systems. To date, 6 such systems are known. Their main characteristics are summarized in Table 1. A few cases, like the resonant planets around HD 82943 [17] - see Fig 4 - and Gl 876 [14], or the planet-brown dwarf pair around HD 168443 [27], are of particular interest; their orbital configu-

Table 1. Characteristics of the known multi-planetary Systems.

\begin{tabular}{lcccccccc}
\hline Star & $\begin{array}{c}\mathrm{P}_{1} \\
{[\text { days }]}\end{array}$ & $\begin{array}{c}\mathrm{P}_{2} \\
\text { days }]\end{array}$ & $\begin{array}{c}\mathrm{P}_{3} \\
{[\text { days }]}\end{array}$ & $\begin{array}{c}\mathrm{m}_{1} \\
{\left[\mathrm{M}_{\mathrm{J}} / \sin i\right]}\end{array}$ & $\begin{array}{c}\mathrm{m}_{2} \\
{\left[\mathrm{M}_{\mathrm{J}} / \sin i\right]}\end{array}$ & $\begin{array}{c}\mathrm{m}_{3} \\
{\left[\mathrm{M}_{\mathrm{J}} / \sin i\right]}\end{array}$ & $\begin{array}{c}\text { Ref. for } \\
\text { First Planet }\end{array}$ & $\begin{array}{c}\text { Ref. for } \\
\text { System }\end{array}$ \\
\hline \hline$v$ And & 4.62 & 241 & 1308 & 0.71 & 2.11 & 4.61 & {$[4]$} & {$[3]$} \\
HD 83443 & 2.98 & 29.8 & - & 1.14 & 0.53 & - & {$[18]$} & {$[18]$} \\
HD 168443 & 58.1 & 1667 & - & 7.2 & 15.1 & - & {$[15]$} & {$[28]$} \\
G1 876 & 61.02 & 30.1 & - & 1.89 & 0.56 & - & {$[5],[16]$} & {$[14]$} \\
HD 82943 & 444 & 221 & - & 1.63 & 0.88 & - & {$[19]$} & {$[17]$} \\
HD 74156 & 51.61 & 2300 & - & 1.56 & $>7.5$ & - & {$[17]$} & {$[17]$} \\
\hline
\end{tabular}



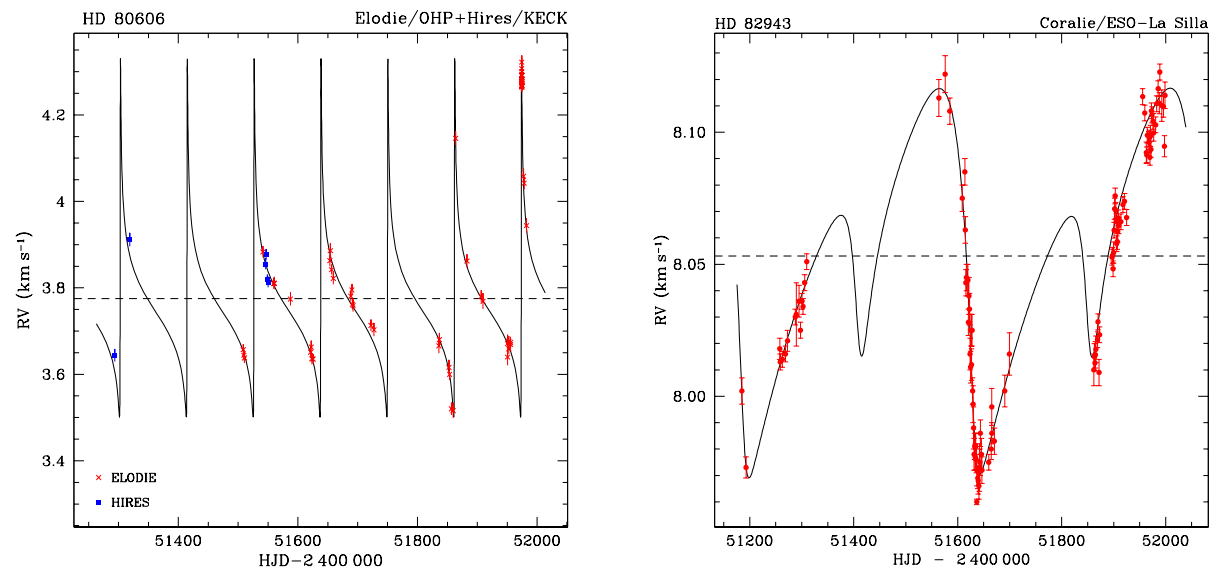

Fig. 4. Left: Radial-velocity measurements and best Keplerian solution for HD 80606. The discovered planet orbits the star in a highly eccentric orbit (e 0.93) with a period of 111.78-days [21]; Right: the same for HD 82943, a system of two resonant planets [17].

rations may provide new constraints on the planetary migration and eccentricity pumping mechanisms (cf. [1]).

\section{The Metallicity Correlation}

One of the most promising results that became evident after the discovery of the first exo-planets is that their host stars have shown to be very metal-rich when compared with dwarf stars in the solar neighborhood [8], [26]. In Fig.5 (left) we can see a comparison between the $[\mathrm{Fe} / \mathrm{H}]$ distribution of a volume limited sample of field dwarfs without planetary mass companions, and the same distribution for the stars with planets [26]. There is a remarkable difference between both distribution, as can be seen from their cumulative functions Fig. 5 (right).

There are basically two ways of interpreting this result. The first is saying that the $[\mathrm{Fe} / \mathrm{H}]$ excess is the result of the accretion of planets and/or planetary material into the star. The second, is to consider that the planetary formation mechanism is dependent on the metallicity of the proto-planetary disk: according to the "traditional" view, a gas giant planet is formed by runaway accretion of gas by a $\sim 10$ earth masses planetesimal. The higher the metallicity (and thus the number of dust particles) the faster a planetesimal can growth, and the higher the probability of forming a giant planet before the gas in the disk dissipates.

Recent results seem to support the latter scenario [26]. The argument is mostly based on the fact that material falling into a star's surface would induce a different increase in $[\mathrm{Fe} / \mathrm{H}]$ depending on the stellar mass, i.e. on the depth of its convective envelope (where mixing can occur). However, the data shows no such trend. Furthermore, the results also show that the $[\mathrm{Fe} / \mathrm{H}]$ distribution of stars with planets steeply rises for higher metallicities, which might be interpreted as 

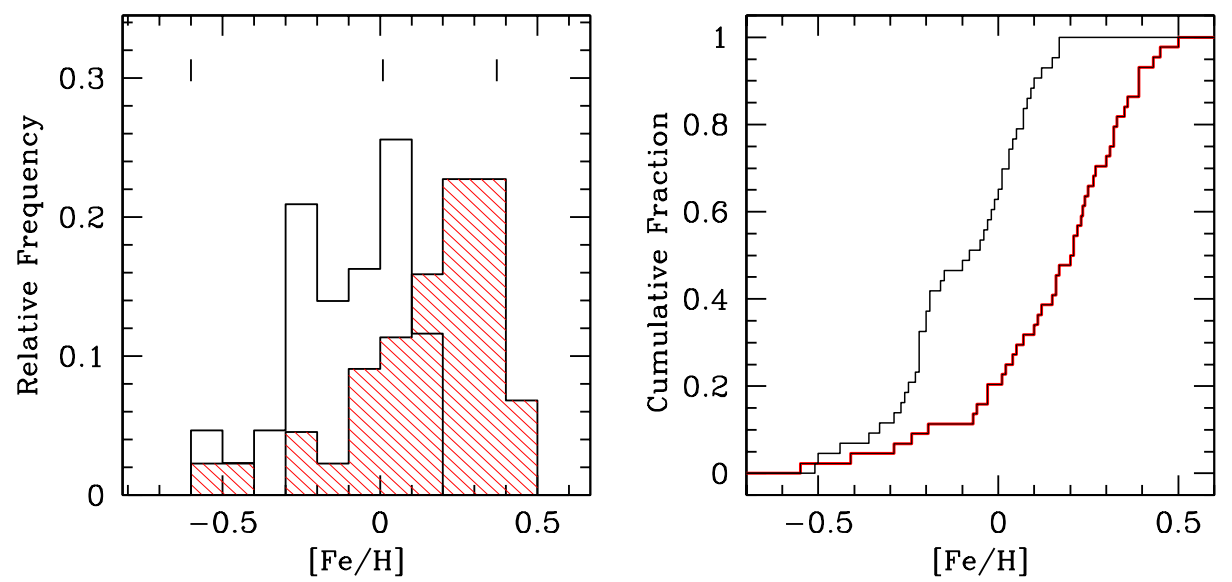

Fig. 5. Left: $[\mathrm{Fe} / \mathrm{H}]$ distribution of stars with planets (shaded histogram) compared with the same distribution of field dwarfs in the solar neighborhood (open histogram) [26]. The vertical lines represent stars with brown dwarf candidate companions having minimum masses between 10 and $20 \mathrm{M}_{\mathrm{Jup}}$. Right: The cumulative functions of both samples. A Kolmogorov-Smirnov test shows the probability of the stars' being part of the same sample is around $10^{-7}$.

an evidence that the planet formation mechanisms are highly dependent on the metallicity of the disk. Our own Sun is in the "metal-poor" tail of the planet hosts $[\mathrm{Fe} / \mathrm{H}]$ distribution!

These results do not exclude, however, that pollution may play a role (eventually important in some cases), but rather that it is not the key process leading to the observed high-metallicity of the planet host stars. In fact, the recent detection of ${ }^{6} \mathrm{Li}$ in the atmosphere of the star HD 82943 [11] (known to harbor a system of two planets [17]) is most likely an indication that this star has engulfed a planet sometime during its lifetime.

\section{Conclusions and Future Perspectives}

The results presented above are giving astronomers a completely different view on the formation and evolution of the planetary systems. We no longer have the Sun as the only example, and today we have to deal with the peculiar characteristics of the "new" extra-solar planets: a huge variety of periods, eccentricities, masses. After only 5 years, we can say that at least $5 \%$ of the solar type dwarfs have giant planetary companions. Furthermore, one interesting conclusion can be taken: our Solar System, with giant planets orbiting away from the star in quasi-circular orbits, is definitely not typical when compared to the presently discovered extra-solar giant planets. The question now is: is it really untypical or are these systems the exception? To help answer this question several projects are currently in the pipeline. 


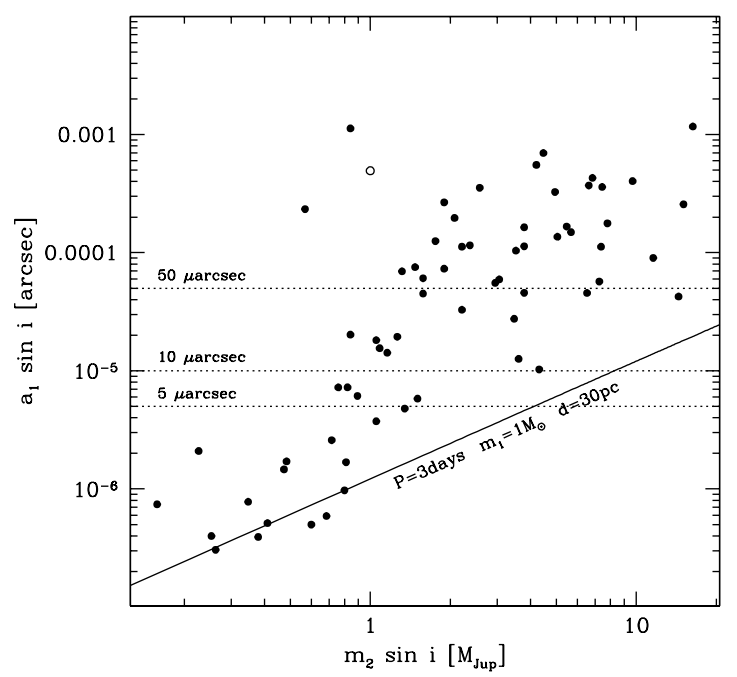

Fig. 6. Mass vs. astrometric motion diagram for stars with very low mass companions discovered by radial-velocity surveys. The dotted lines represent the limits for astrometric precisions of 50,10 and $5 \mu$ arcsec. The open circle illustrates the position of the Sun as seen from a distance of $10 \mathrm{pc}$. The solid line indicates an approximate limit imposed by the fact that no planets were found with periods shorter than $\sim 3$ days.

From radial-velocity searches, the current surveys, including between 2000 and 3000 stars, will continue to increase the number of known exo-planets. Several dozens are expected to be announced in the next few years. On the other hand, the ever increasing precision will permit to discover lighter planets, as well as to increase the number of known multi-planetary systems. In this context, newly or soon available ESO instruments (e.g. UVES/VLT or HARPS/3.6$\mathrm{m}[23])$ will certainly play an important role.

From the astrometric point of view, the expectations are not lower. Instruments like the VLTI or KeckI will give us the possibility to estimate real masses for many of the known planetary systems (see Fig. 6). Furthermore, space missions like GAIA or the interferometric mission SIM will completely change the current landscape by adding tens of thousands of new planets. Given that astrometry is more sensitive to longer period systems (contrary to the radialvelocity method), these projects will also permit to better cover the period distribution of the exo-planets. It will further permit to find planets around targets not accessible with radial-velocity surveys, like A or B stars, or T Tauri stars.

Further hopes will come from photometric transit searches, mostly based upon space missions like COROT, Eddington or Kepler. Out of the Earth's atmosphere, these satellites will achieve a photometric precision better than $0.01 \%$, permitting the detection of transiting earths.

All these steps will permit to better understand the mechanisms leading to the formation of planetary systems like our own, and will thus somehow represent 
an important step towards the search for life in the universe. Two similar projects are currently directed towards this specific goal (Darwin/ESO and TPF/NASA). Using nulling interferometry techniques (to remove the light from the brighter star and leave the one coming from the planet), they will try to find traces of life in the spectrum of exo-earths. In a very close future humanity has to prepare itself to find out that the whole universe may be teeming with life.

We would like to thank the members of the Geneva extra-solar planet search group, D. Naef, F. Pepe, D. Queloz, S. Udry, our French colleagues J.-P. Sivan, C. Perrier and J.-L. Beuzit, as well as G. Israelian and R. Rebolo (from the IAC), who have largely contributed to the results presented here. We wish to thank the Swiss National Science Foundation (FNSRS) for the continuous support to this project. Support from Fundação para a Ciência e Tecnologia, Portugal, to N.C.S. in the form of a scholarship is gratefully acknowledged.

\section{References}

1. Armitage P.J., Livio M., Lubow S.H., Pringle J.E., 2001, submitted to ApJL

2. Bodenheimer P., Hubickyj O., Lissauer J.J., 2000, Icarus 143, 2

3. Butler R.P., Marcy G.W., Fischer D., et al., 1999, ApJ 526, 916

4. Butler R.P., Marcy G.W., Williams E., et al., 1997, ApJ 474, L115

5. Delfosse X., Forveille T., Mayor M., et al., 1998, A\&A 338, L67

6. Fischer D.A., Marcy G.W., Butler R.P., et al., 2001, ApJ 551, 1107

7. Goldreich P., Tremaine S., 1980, ApJ 241, 425

8. Gonzalez G., 1998, A\&A 334, 221

9. Halbwachs J.-L., Arenou F., Mayor M., Udry S., Queloz D., 2000, A\&A 355, 573

10. Han I., Black D., Gatewood G., 2001, ApJ 548, L57

11. Israelian G, Santos N.C., Mayor M., Rebolo R., 2001, Nature 411, 163

12. Jorissen, Mayor M., Udry S., 2001, A\&A, submitted

13. Lin D., Bodenheimer P., Richardson D.C., 1996, Nat. 380, 606

14. Marcy G.W., Butler R.P., Fischer D., et al., 2001 ApJ, in press

15. Marcy G.W., Butler R.P., Vogt S., et al., 1999, ApJ 520, 239

16. Marcy G.W., Butler R.P., Vogt S., 1998, ApJ 505, L147

17. Mayor M., et al., 2001, ESO press-release 07/01

18. Mayor M., Naef F., Pepe F., et al., 2001. In: Penny A., Artimowicz P., Lagrange A.-M., Russel S., "Planetary Systems in the Universe: Observations, Formation and Evolution", ASP Conf. Ser., in press

19. Mayor et al., 2000, ESO press-release 13/00

20. Mayor M., Queloz D., 1995, Nature 378, 355

21. Naef D., et al., 2001, A\&A Letters, submitted

22. Papaloizou J.C., Nelson R.P., Masset F., 2001, A\&A 366, 263

23. Pepe F., Mayor M., Delabre B., et al., 2000, Proc. SPIE Vol. 4008, 582

24. Pourbaix D., Arenou F., 2001, A\&A, in press

25. Rasio F.A., Ford E.B., 1996, Science 274, 954

26. Santos N.C., Israelian G., Mayor M., 2001, A\&A, in press

27. Udry S., Mayor M., Halbwachs J.-L., Arenou F., 2000, in "Microlensing 2000", Eds. Menzies J.W., Sackett P.D., ASP Conf. Series, in press

28. Udry S., Mayor M., Queloz D., 2001. In: Penny A., Artimowicz P., Lagrange A.M., Russel S., "Planetary Systems in the Universe: Observations, Formation and Evolution", ASP Conf. Ser., in press 